\title{
CANONICAL FORMS OF SELF-ADJOINT BOUNDARY CONDITIONS FOR REGULAR DIFFERENTIAL OPERATORS OF ORDER THREE
}

\author{
Tian Niu, Xiaoling HaO, Jiong Sun and Kun Li
}

\begin{abstract}
In this paper, we find all canonical forms for third order self-adjoint boundary conditions. These canonical forms play an important role in the study of the dependence of the eigenvalues on the problem and for their numerical calculation. In order to obtain those canonical forms, we give a classification of self-adjoint boundary conditions. Those self-adjoint boundary conditions can be categorized into three mutually exclusive classes: coupled, strictly separated and mixed. Unlike the even order case, for the third order case, the strictly separated self-adjoint boundary conditions can not be realized. For coupled and mixed cases, there are some different types for the canonical forms: 2 for coupled and 4 for mixed boundary conditions.
\end{abstract}

Mathematics subject classification (2010): 34B24, 34L15.

Keywords and phrases: Canonical forms, differential operators, boundary conditions.

\section{REFERENCES}

[1] Zettl, A., Sturm-Liouville Theory, Providence, Rhode Island: American Mathematics Society, Mathematical Surveys and Monographs, 121, 2005.

[2] Zettl, A., Sun, J., Survey article: Self-adjoint ordinary differential operators and their spectrum, Rocky Mountain Journal of Mathematics, 2015, 45(3):763-886.

[3] Kong, Q, Zettl, Q., Dependence of eigenvalues of Sturm-Liouville problems on the boundary, Journal of Differential Equations, 1996, 126(2):389-407.

[4] HaO, X., Sun, J., ZETTL, A., Canonical forms of self-adjoint boundary conditions for differential operators of order four, Journal of Mathematical Analysis and Applications, 2012, 387(2):1176-1187.

[5] SuO, J., WANG, W., Eigenvalues of a class of regular fourth-order Sturm-Liouville problems, Appl. Math. Comput., 2012, 218:155-168.

[6] Zhang, M., Wang, Y., Dependence of eigenvalues of Sturm-Liouville problems with interface conditions, Appl. Math. Comput., 2015, 265:31-39.

[7] LI, K., SUn, J., HaO, X., Eigenvalues of regular fourt order Sturm-Liouville problems with transmission conditions, Mathematical Methods in the Applied Sciences, 2017, 40:3538-3551.

[8] HaO, X., Zhang, M., Sun, J., Characterization of domains of self-adjoint ordinary differential operators of any order, even or odd, Electronic Journal of Qualitative Theory of Differential Equations, 2017, 2017(61):1-19.

[9] Naimark, M.A., Linear differential operators, Ungar, New York, 1968.

[10] WANG, A., Sun, J., ZETTL, A., The classification of self-adjoint boundary conditions: separated, coupled, and mixed, Journal of Functional Analysis, 2008, 255(6):1554-1573. 